\title{
The negative effect of smartphone use on academic performance may be overestimated: evidence from a two-year panel study
}

\author{
Andreas Bjerre-Nielsen ${ }^{\mathrm{a}, \mathrm{b}, *}$, Asger Andersen ${ }^{\mathrm{a}}$, Kelton Minor $^{\mathrm{a}}$, David Dreyer Lassen $^{\mathrm{a}, \mathrm{b}, \mathrm{c}}$ \\ ${ }^{a}$ Copenhagen Center for Social Data Science, University of Copenhagen \\ ${ }^{b}$ Department of Economics, University of Copenhagen \\ ${ }^{c}$ Center for Economic Behavior and Inequality, University of Copenhagen
}

\begin{abstract}
This study monitored 470 university students' smartphone usage continuously over two years to assess the relationship between in-class smartphone use and academic performance. We utilize a novel dataset where smartphone use and grades were recorded across multiple courses, allowing us to examine this relationship at the student level and the student-incourse level. In accordance with the existing literature, we find that students' in-class smartphone use is negatively associated to their grades, even when controlling for a broad range of observed student characteristics. However, the magnitude of the association decreases substantially in a fixed effects model, which leverages the panel structure of the data to control for all stable student and course characteristics, including those not observed by researchers. This suggests that the size of the effect of smartphone usage on academic performance is overestimated by studies that only control for observed student characteristics. Keywords: academic performance, attention, mobile devices, multitasking, in-class concentration, productivity, distraction
\end{abstract}

* Corresponding author

Email addresses: andreas.bjerre-nielsen@econ.ku.dk (Andreas Bjerre-Nielsen), asa@sodas.ku.dk (Asger Andersen), kmi@samf.ku.dk (Kelton Minor), david.dreyer.lassen@econ.ku.dk (David Dreyer Lassen)

$U R L:$ https://abjer.github.io (Andreas Bjerre-Nielsen) 


\section{Introduction}

Smartphones have become pervasive in learning environments. A recent survey study found that $96 \%$ of US college students own a smartphone (Brooks and Pomerantz, 2017) and additional evidence suggests that in-class mobile device use has become common (Felisoni and Godoi, 2018; Ravizza et al., 2017). These devices enable students to engage in potentially learning-enhancing activities, such as taking notes, participating in online quizzes or looking up pertinent facts on the internet. However, the very same devices also connect students to an enticing menu of non-academic stimuli that may distract from the learning processes taking place in their immediate learning environments (Stokols, 2018). Aside from attention, device use may also inhibit other cognitive pathways involved in learning, including memory and reward processing (Chen and Yan, 2016; Uncapher and Wagner, 2018; Wilmer et al., 2017). However, recent research also emphasizes that the results of studies on the effects of digital device use might depend heavily on the usage context from which the data is gathered and the subsequent methods of analysis chosen by the researchers (Orben and Przybylski, 2019; Orben et al., 2019; Adelantado-Renau et al., 2019; Whitlock and Masur, 2019).

A nascent body of experimental and observational research has started to investigate the impact of portable device use on student learning within the classroom setting using both self-reported and directly recorded measures of use. Experimental studies based on brief interventions have established that in-class multitasking with electronic devices can limit short term knowledge retention (Mendoza et al., 2018; Risko et al., 2010; Wood et al., 2012). Due to the short testing periods and possible presence of experimental demand characteristics, these experiments do not necessarily generalize to the real-world learning environments and longer evaluation cycles found in educational institutions. Observational studies, while unable to claim causality, overall find similar negative links between portable device use and academic performance (Grace-Martin and Gay, 2001; Kraushaar and Novak, 2010; Fried, 2008; Kirschner and Karpinski, 2010; Ravizza et al., 2017; Lepp et al., 2014; Uzun and Kilis, 2019; Kim et al., 2019).

The majority of prior observational studies have relied heavily on self-reported or self- 
initiated measures of device use. However, self-reports of digital device use have been found to be inaccurate, exhibiting little relation to true usage (Kraushaar and Novak, 2010; Andrews et al., 2015; Kim et al., 2019; Orben and Przybylski, 2019). Only a small subset of the observational studies measured usage directly by digitally tracking student device behaviors, and did so only for a few weeks, a single course and/or over a single term (Grace-Martin and Gay, 2001; Kraushaar and Novak, 2010; Ravizza et al., 2017; Felisoni and Godoi, 2018). Participants in (Ravizza et al., 2017) were required to manually activate an online web activity logger at the start of every class. This approach overcomes problems of self-reporting, but may inadvertently prime students by periodically drawing their attention to their device use at the start of every class measurement period. Self-activated tracking may also bias participation in the study through selective logging and errors of omission. By comparison, the recent advent of smartphone activity tracking apps provides a less invasive approach to collect device interactions in the background (Felisoni and Godoi, 2018; Kim et al., 2019).

Existing studies have focused on the relationship between academic performance and device use during a single course or collected average measurements across several courses (Chen and Yan, 2016). Since these cross-sectional studies only include a single row of measurements for each participating student, they cannot control for unobserved variables that might jointly determine device use and academic performance. While some studies directly measure and control for specific student traits that could confound the estimated effects - including intelligence, motivation and interest (Ravizza et al., 2014, 2017) - other student characteristics may act as confounding factors. For instance, students with low levels of self-control may use their phone more often in class, study less intensively outside of class, and perform worse on academic outcomes, with phone use a symptom of limited self-control rather than a cause of lower grades (Wilmer and Chein, 2016). Likewise, grades and smartphone use may jointly reflect contextual factors at the course level such as topic, instructor, room and class characteristics, which may limit the external validity of prior research reliant on single course observations.

In the present study, we used a mobile app to unobtrusively log students' complete attendance and smartphone use across multiple courses over a period spanning two aca- 
demic years. This enables us to examine the link between students' average smartphone use and average grade, as well as the relationship between students' course-specific use and performance. Additionally, we combine this data with a wide range of individual background controls, including administrative data on high school academic performance, socioeconomic background and surveyed personality measures. We provide a novel measure of in-class smartphone use and employ this to investigate the primary hypothesis that higher non-academic device use during class is associated with worse student performance. We expected to find a moderate to large negative relationship between students' smartphone use and grades both when estimating with a cross-sectional model, consistent with the existing literature, and with a fixed effects model that leverages the dynamic nature of our panel data to control for unobserved student and course characteristics.

\section{Method}

\section{Participants}

We collected data from September 2013 until September 2015 as part of the Copenhagen Networks Study (Stopczynski et al., 2014). Our study used data from 470 students at the Technical University of Denmark (DTU). Students volunteered to receive a smartphone that continuously recorded various behavioral measures over a two year period, including location, social interactions among participants and whether the phone screen was turned on. The study was approved by the Danish Data Supervision Authority in 2013 and involved dynamic informed consent. After consenting to participate in the study, students had access to download their own logged data and could withdraw from the study and have their data deleted at any time (see Stopczynski et al., 2014). The data collection period was planned to terminate after two years due to the anticipated costs of maintaining data collection, replacing broken phones and attrition over time. The size of our sample far exceeds earlier observational studies using smartphones both in terms of individuals followed and data points per individual (Felisoni and Godoi, 2018; Kim et al., 2019). Table 1 displays descriptive statistics for the 470 students meeting the inclusion criteria outlined below, at the start of the study in September 2013. 
Table 1. Descriptive statistics - collected in September 2013 - for the 470 students in the sample. See more about the variables in the section Student background variables.

\begin{tabular}{lrrrrrrr}
\hline Variable & Min & 1st quartile & Median & 3rd quartile & Max & Mean & SD \\
\hline Age & 19.0 & 20.0 & 21.0 & 21.5 & 28.8 & 21.0 & 1.6 \\
Danish high school grade average & 3.8 & 7.3 & 8.6 & 9.6 & 11.7 & 8.4 & 1.8 \\
Parents' max years of education & 9.8 & 14.2 & 15.0 & 17.0 & 20.0 & 15.3 & 2.4 \\
Parents' mean income & 12.5 & 37.4 & 46.6 & 58.5 & 183.0 & 50.0 & 22.8 \\
Agreeableness & 2.2 & 3.5 & 3.9 & 4.1 & 4.8 & 3.8 & 0.5 \\
Conscientiousness & 2.0 & 3.0 & 3.4 & 3.9 & 4.8 & 3.4 & 0.6 \\
Extraversion & 1.7 & 3.0 & 3.4 & 3.9 & 4.8 & 3.4 & 0.7 \\
Neuroticism & 1.1 & 1.9 & 2.4 & 2.8 & 4.1 & 2.4 & 0.6 \\
Openness & 2.1 & 3.2 & 3.6 & 3.9 & 4.8 & 3.5 & 0.5 \\
Locus of control & 2.0 & 6.0 & 8.0 & 9.0 & 12.2 & 7.8 & 2.2 \\
BMI & 16.5 & 20.7 & 22.4 & 24.0 & 34.8 & 22.7 & 3.0 \\
\hline Male (1 if male; 0 if female) & - & - & - & - & - & 0.78 & - \\
Smoker (1 if smoker; 0 if non-smoker) & - & - & - & - & - & 0.25 & - \\
\hline
\end{tabular}

Note: Due to data privacy reasons each quantile is calculated as the average of the 5 observations around the actual quantile. 
Table 2. Descriptive statistics for the variables collected during the study.

\begin{tabular}{lrrrrrrr}
\hline Variable & Min & 1st quartile & Median & 3rd quartile & Max & Mean & SD \\
\hline Smartphone use in-class & 0.1 & 3.9 & 6.6 & 11.0 & 41.1 & 8.2 & 6.1 \\
Avr. smartphone use in-class & 1.0 & 4.9 & 7.4 & 11.1 & 28.7 & 8.6 & 5.3 \\
Smartphone use out-of-class & 1.0 & 5.8 & 7.7 & 10.7 & 20.8 & 8.3 & 3.8 \\
Course grades & -3 & 4 & 7 & 10 & 12 & 7.3 & 3.8 \\
Grade average & -1.6 & 5.1 & 7.2 & 9.2 & 12.0 & 6.9 & 2.9 \\
\hline
\end{tabular}

Note: The Smartphone use in-class, Grades and Smartphone use out-of-class variables are measured on the student-in-course level (3385 observations). The Avr. smartphone use in-class and Grade average variables are measured on the student level (470 observations). Due to data privacy reasons each quantile is calculated as the average of the 5 observations around the actual quantile.

Students in the Copenhagen Networks Study were excluded from the analysis if they met any of the following criteria: no grade data (76 students); less than 10 hours of course class attendance (60 students); missing background variables (157 students); enrollment in only one graded course (44 students) or in courses with no other participants of our study (3 students). After applying these criteria to the initial sample of 810 students, 340 students were excluded. Additional details of the data filtering process are described in the Supplementary Online Material [SOM], where we also confirm that our results are robust to including the students with only little class attendance and the students with missing background variables in the analysis.

\section{Measures}

Smartphone use. For each student we computed a measure of phone usage at the 15 minute level by aggregating the time the screen of the student's phone was turned on during each 15 minute time bin of the experiment. We also measured each student's attendance in scheduled classes by merging administrative data from DTU about the location of the student's scheduled classes with mobile device geolocation data; see details about how we 
inferred attendance in (Kassarnig et al., 2017). We combined these two measures to compute smartphone use in-class. This variable measured - for each course a student was enrolled in - the percentage of attended class time that the student spent with his/her phone screen turned on (see the supplementary online material for details about how we addressed breaks between classes). As an example, let $s_{i j}$ denote the value of smartphone use in-class for student $i$ in course $j$ : If $s_{i j}=5$, it means that student $i$ 's phone screen was turned on 5 percent of the time they attended classes in course $j$. In our cross-sectional analysis, we employed each student's average smartphone use in-class across all attended courses as our measure of smartphone use. We also computed Smartphone use out-of-class, which measured the percentage of time between 6am and 1am, where a student's mobile device screen was turned on and the student was not attending class. Table 2 displays descriptive statistics for the smartphone use measures.

When activated by an event, such as receiving an SMS or pressing a button, smartphones have a default period of time before the screen turns off. Our smartphone use measure could therefore include time intervals where the student's attention was not directed towards the phone, but the screen was still turned on. Our data suggests that the most prevalent default turn-off times are 10 and 30 seconds; see details in the SOM. Since almost all of the smartphone use in our sample was spent in sessions longer than 35 seconds and intermittent periods of screen activation can still captivate attention, we assume that our measure of smartphone use exclusively recorded time when the student was attending to their phone.

We did not observe what kind of phone activity the students engaged in. Thus, our smartphone use measure did not divide device activity into academic and non-academic use. Although this is a limitation of the tracking app used in this study, prior research indicates that the majority of in-class device use typically consists of non-academic activities such as texting and social media (Gehlen-Baum and Weinberger, 2014; Ravizza et al., 2017; Appel et al., 2019). Furthermore, in contrast to the online activity measures used in previous studies, our measures capture all smartphone screen exposure, including both online and offline (ex. app-based and system based) mobile device interactions, all of which can occupy attention. 
Academic performance. We obtained the students' course grades and study programs from university administrative records. Grades were given on the Danish grading scale and consisted of 7 possible values: $-3,0,2,4,7,10$ and 12. In our cross-sectional analysis, we used each student's average grade across all courses, whereas the panel analysis employed the course-specific grades. Table 2 displays descriptive statistics for the course grades and grade averages.

Student background variables. We obtained various student characteristics for our sample by merging it with two sources. First, we obtained surveyed personality measures (the Big Five Inventory [BFI] and locus of control) and health status (Body Mass Index [BMI] and whether the student smoked cigarettes) from the Copenhagen Network Study itself. BFI and locus of control were measured with the standard survey items administered at the beginning of the study period (Goldberg, 1993; Rotter, 1993). The distribution of personality traits in the Copenhagen Network Study was previously shown to be unbiased with regards to the general population of Western Europe (Stopczynski et al., 2014). Second, this data was pseudonymized and merged with national registries on secure servers with limited access at Statistics Denmark. Here, we obtained student age, gender and high school grade averages as well as mean annual income and maximum number of years of education of students' parents. Parental mean annual income was measured in units of 10,000 DKK. As a proxy for intelligence, high school grade averages were computed from grades on the same scale as the university grades (Roth et al., 2015).

\section{Structure of the data}

The filtered data had 3385 observations, each containing the grade, in-class smartphone use and student background variables of a specific student in a specific course. Each observed student participated in multiple courses, and each observed course had multiple students who were part of our experiment. However, each student did not participate in all observed courses, and neither did each course have all observed students as participants. Thus, our data was structured as an unbalanced panel (Wooldridge, 2010). The median number of 
Table 3. Panel model specifications

\begin{tabular}{lll}
\hline \multicolumn{1}{c}{ Specification } & Control variables \\
\hline (1a) & $g_{i j}=\alpha+\beta s_{i j}+\epsilon_{i j}$ & None \\
\hline (1b) & $g_{i j}=\alpha+\beta s_{i j}+\gamma^{t} \mathbf{x}_{i}+\epsilon_{i j}$ & Observed student background variables \\
\hline (1c) & $g_{i j}=\alpha+\beta s_{i j}+\gamma^{t} \mathbf{x}_{i}+\eta_{j}+\epsilon_{i j}$ & Observed student background variables and course fixed effects \\
\hline (1d) & $g_{i j}=\alpha+\beta s_{i j}+\mu_{i}+\epsilon_{i j}$ & Student fixed effects \\
\hline (1e) & $g_{i j}=\alpha+\beta s_{i j}+\mu_{i}+\eta_{j}+\epsilon_{i j}$ & Student and course fixed effects \\
\hline
\end{tabular}

Note: For all model specifications we used two-way clustering (Cameron and Miller, 2015) to cluster standard errors at both the student and course level, see Models for details.

recorded courses per student was 7 and the median number of observed students per course was 6 . The data contained 470 unique students and 401 unique courses.

We used our panel data to construct cross-sectional data by averaging each student's grades and in-class smartphone use across all the student's courses. The resulting data set had 470 observations, each containing the average grade, average smartphone use in-class and background variables of a specific student. We refer to models estimated on the panel data set as panel models, whereas we refer to the models estimated on the cross-sectional data set as cross-sectional models. An advantage of the panel models relative to crosssectional models is that they allow for estimation of within-students effects instead of only between-student effects (Orben et al., 2019).

\section{Sample size determination}

Our data was collected as part of the longitudinal Copenhagen Networks study (Stopczynski et al., 2014), which was conducted prior to the formulation of our research question. Thus, similar to other recent research employing digital traces from large behavioral datasets (Rafaeli et al., 2019), our sample size was not predetermined by our research question or expected effect size. However, compared to previous similar observational studies (see Introduction) the sample size and observation period in the present study were respectively larger and longer. Moreover, we did select our models and construct our variables prior to seeing the results of the models. 
Table 4. Correlation coefficients between measures of in-class multitasking and measures of academic performance from previous observational studies.

\begin{tabular}{|c|c|c|c|c|c|}
\hline Cite & Design & Sample size & Device(s) & Outcome & $r$ \\
\hline Fried (2008) & Survey & 137 & Laptop & Exam score & -0.17 \\
\hline Karpinski et al. (2013) [EU] & Survey & 406 & Laptop \& Phone & GPA & -0.27 \\
\hline Karpinski et al. (2013) [US] & Survey & 451 & Laptop \& Phone & GPA & -0.60 \\
\hline Lepp et al. (2014) & Survey & 496 & Phone & GPA & -0.20 \\
\hline Ravizza et al. (2017) & Field study & 61 & Laptop & Exam score & -0.25 \\
\hline Felisoni and Godoi (2018) & Field study & 43 & Phone & Exam score & -0.31 \\
\hline Our study & Field study & 470 & Phone & GPA & -0.32 \\
\hline
\end{tabular}

\section{Models}

Models using panel data. Table 3 displays the specifications of the models we have estimated on our panel data. The grade and smartphone use in-class of student $i$ in course $j$ are denoted by $g_{i j}$ and $s_{i j}$. All the models estimate linear associations between course grades and smartphone use, but each model controls for a different set of additional explanatory variables. We consider Model 1e to provide the most accurate estimate of the linear effect of in-class smartphone use on grades, since the student and course specific intercepts $\mu_{i}$ and $\eta_{j}$ control for all student and course characteristics that remained fixed during the study. Examples of such student characteristics are gender, age at baseline, intelligence, stable personality traits, impulsivity, persistent anxiety, and the socioeconomic conditions of the student's upbringing. Examples of such course characteristics include quality of the teacher, difficulty of the course material, class size and room layouts. We therefore consider Model 1e to be our main model, and we only estimate the other models to enhance our understanding of it. In this section, we therefore first present Model 1e, and then we explain why each of the other models are interesting as points of comparison. 
Model 1e is an example of a type of panel model called fixed effects models (McNeish and Kelley, 2019; Wooldridge, 2010), and the student and course specific intercepts $\mu_{i}$ and $\eta_{j}$ are called the student and course fixed effects. Estimation is possible with a standard linear regression, where $\mu_{i}$ and $\eta_{j}$ are estimated by encoding each student and course as a dummy variable. The main advantage of a fixed effects model is consistent estimation of model parameters under weaker assumptions about the data, whereas mixed effects models, traditionally more prevalent in psychological research for clustered data, rely on stronger statistical assumptions in order to be consistent (McNeish and Kelley, 2019; Wooldridge, 2010). The key additional assumption of mixed effects models is the exogeneity assumption, which requires that the student and course specific intercepts are random and thus uncorrelated with smartphone use in-class (McNeish and Kelley, 2019; Wooldridge, 2010). This assumption is more justifiable in experimental studies, where the variable of interest can be random by design. However, in observational studies such as ours this strong assumption is likely to be violated (McNeish and Kelley, 2019), since unobserved individual and course characteristics are almost certainly correlated with smartphone use in-class. For example, low teacher quality, which is unobserved, could affect both smartphone use and grades. Indeed, we view the strength of our panel data approach to reside in the fact that it can control for such confounding student and course characteristics even when they are unobserved. In addition to these theoretical considerations, our choice of a fixed effects model over a mixed effects model is also supported by a Hausman specification test $\left(\chi^{2}(1, N=3385)=8.48, p=0.004\right)$, which is the standard statistical test used to assess the consistency of a null model with random effects against the corresponding fixed effects model (Wooldridge, 2010).

To make our results comparable with the existing observational studies, we estimated Model 1b which is a pooled model. This model is simply a linear regression of course grade on observed student characteristics, similar to the cross-sectional model below but with an observation per student-in-course combination (thus multiple observations per student). Therefore, Model 1b ignores the panel structure of the data and thus has neither mixed nor fixed effects. The vector $\mathbf{x}_{i}$ in the model specification (see Table 3 ) contains the values 

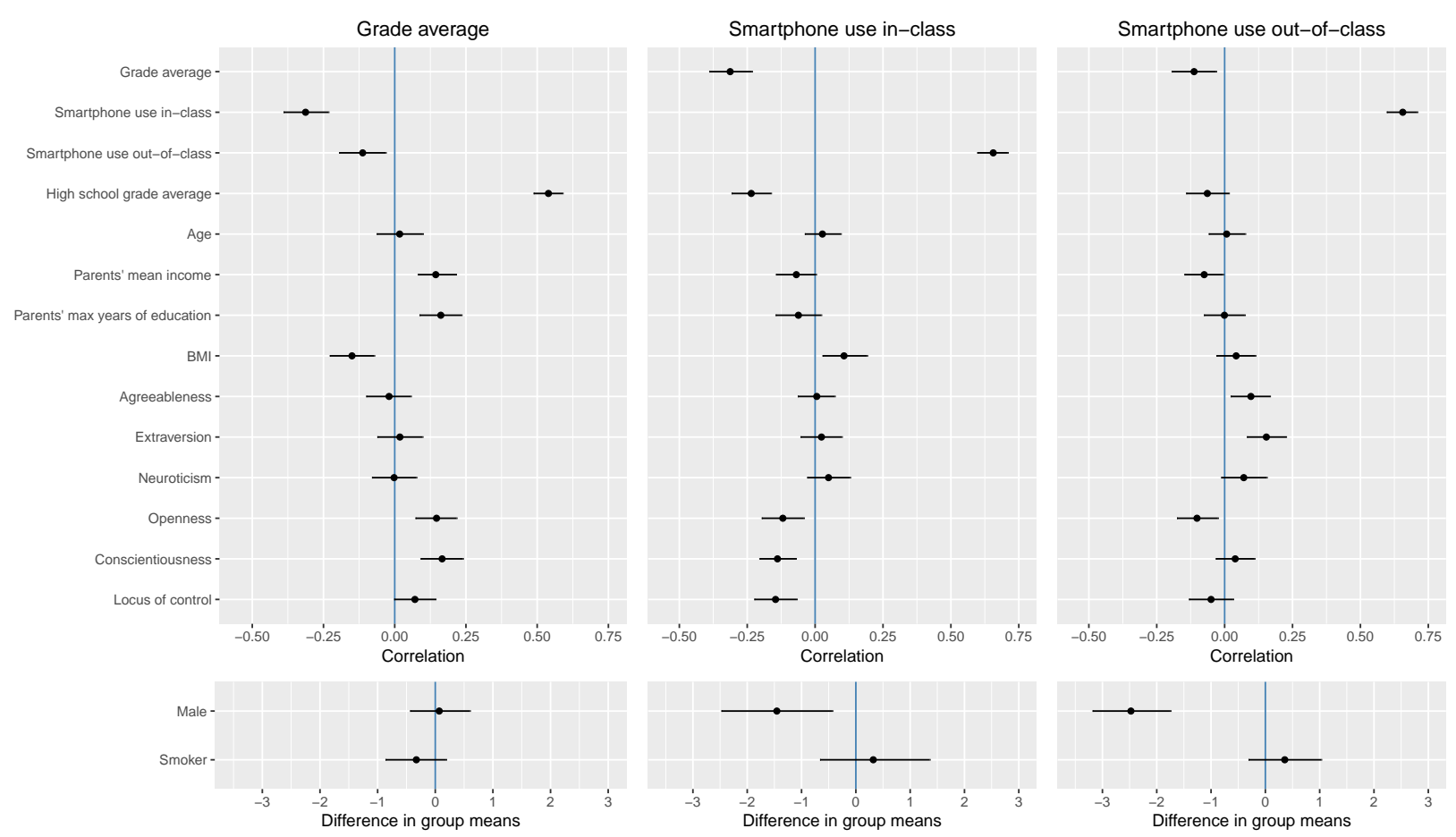

Fig. 1. Correlations between average grade, average smartphone use in-class and the student background variables. The dots mark the observed correlations, and the lines mark the $95 \%$ confidence intervals. We calculated the confidence intervals by bootstrapping the data 10,000 times, calculating the pairwise correlations on each of the bootstrapped samples and taking the $5^{\text {th }}$ and $95^{\text {th }}$ percentile as the endpoints of the intervals. The top plots display the numeric variables, whereas the bottom ones show the categorical factors.

of the observed background variables ${ }^{1}$ for student $i$. Similar to the existing observational studies, this model only controls for observed student characteristics when assessing the association between device use and academic performance. Consequently, its estimate of $\beta$ is inconsistent if grades and smartphone use in-class are confounded by variables that are not measurable by the researcher (Wooldridge, 2010). Substantial differences between the estimates of $\beta$ in Model $1 \mathrm{~b}$ and Model 1e thus suggest that there exist important confounding factors that are not captured by our background variables.

We estimated Models 1a, 1c and 1d to investigate how the estimated coefficient of smart-

\footnotetext{
${ }^{1}$ Age, gender, high school grade average, parents' mean income and max years of education, measures of 6 personality traits, BMI and whether or not the student smokes; see details in the section Student background variables.
} 
phone use in-class responds to the addition of different controls. Model 1a provides a baseline without any controls. Model 1c controls for observed student characteristics and course fixed effects, but not student fixed effects. Model 1d controls for student, but not course fixed effects.

To account for the fact that two or more data points belong to the same individual and/or course, i.e. the panel structure, we adjusted the standard errors. This is analogous to random effects where standard errors are computed such that they reflect sampling clusters, which in our case are students and courses. We followed (Cameron and Miller, 2015) and used two-way clustering to cluster standard errors at both the student and course levels. This clustering of standard errors accounts for possible correlation of error terms within individuals and courses (Cameron and Miller, 2015).

Cross-sectional model. To make our research comparable with the existing literature, we also estimated a linear regression model with the cross-sectional data (Model 2) which had the following specification:

$$
\bar{g}_{i}=\alpha+\beta \bar{s}_{i}+\gamma^{t} \mathbf{x}_{i}+\epsilon_{i}
$$

where $\bar{g}_{i}$ and $\bar{s}_{i}$ are, respectively, the average grade and smartphone use in-class of student $i$ across all of the courses they attended during the experiment. The vector $\mathbf{x}_{i}$ contains the values of the background variables for student $i$. Consistent with the existing literature, the cross-sectional model only has a single observation for each student in our sample, and the effect of smartphone use in-class on academic performance is estimated controlling for a broad set of student-level background variables.

\section{Results}

We first report correlation measures, proceed with the output of the panel models (Model 1a-1e in Table 3) and finish by comparing these to the output of our cross-sectional model (Model 2). 
Table 5. Estimated association between smartphone use in-class on student course grades.

\begin{tabular}{ccccc}
\hline & Coefficient, $\hat{\beta}$ & $\begin{array}{c}\mathbf{9 5 \%} \text { confidence interval } \\
\text { of coefficient, } \hat{\beta}\end{array}$ & $\begin{array}{c}\text { Standardized } \\
\text { coefficient }\end{array}$ & Adjusted $\mathbf{R}^{2}$ \\
\hline$(1 \mathrm{a})$ & -0.132 & $(-0.165,-0.099)$ & -0.213 & 0.04 \\
\hline$(1 \mathrm{~b})$ & -0.081 & $(-0.110,-0.051)$ & -0.129 & 0.19 \\
\hline$(1 \mathrm{c})$ & -0.072 & $(-0.099,-0.045)$ & -0.116 & 0.38 \\
\hline$(1 \mathrm{~d})$ & -0.046 & $(-0.077,-0.016)$ & -0.074 & 0.43 \\
\hline$(1 \mathrm{e})$ & -0.028 & $(-0.057,0.001)$ & -0.045 & 0.59 \\
\hline
\end{tabular}

Note: The Coefficient column displays the estimate of $\beta$ from each of the panel models. The specifications of the models can be found in Table 3. The Standardized coefficient column displays the estimated $\beta$, when both grades and smartphone use in-class were standardized. For specifications of clustered standard errors, see Models section.

Correlations. To make our study more comparable with prior investigations, we compared our estimated correlation coefficient between students' average smartphone use across courses and grade averages with estimates from earlier observational studies; see Table 4. Our estimated correlation $(r=-0.32)$ is similar in magnitude to most previously found correlations. Its 95 percent confidence interval $(-0.40,-0.24)$ contains four of the six earlier studies. The confidence interval was estimated from 10,000 bootstrap samples.

Figure 1 shows the pairwise correlations between each of the students' grade average, average in-class smartphone use across all attended courses, smartphone use out-of-class and the background variables. We see from the left plot that students' grade average is more strongly correlated with in-class smartphone use than with any of the student background variables, except for high school grade average. This shows that there is a substantial negative association between smartphone use and grade average, although it might not be a causal relationship. We also see from the center plot that smartphone use in-class was almost as strongly correlated with high school grade average as with university grade average. One explanation for this is that students with high smartphone use in university courses may also 
have been heavy smartphone users in high school, which could conceivably have contributed to a lower high school grade average. Another explanation, however, is that both correlations are caused by underlying student characteristics that confound smartphone behaviour and academic performance in both high school and university learning environments. Further, we see from the figure that a number of the background variables were positively correlated with in-class smartphone use but negatively associated with grade average and vice versa. This suggests that at least part of the observed correlation between smartphone use and grade average is explained by confounding factors measured by the subset of background variables that we observe. Finally, we see from the right plot that in-class and out-of-class smartphone use are highly correlated $(r=0.66)$. However, compared to in-class use, outof-class use is a weaker predictor of grade average.

Models using panel data. Table 5 reports the results of our models based on panel data. We see that Model 1e's estimate of $\beta$ is around one-third of the estimate found by Model 1b, and Model 1e's confidence interval for $\beta$ does not include the estimate found by Model $1 \mathrm{~b}$. As explained in the Methods section, this suggests that the estimate found by Model 1b was severely biased due to confounders that were not fully captured by the student background variables. The majority of the bias is explained by the student fixed effects - this is seen in the substantial difference when controlling for all stable student traits (including latent characteristics) compared to only controlling for the measured student background variables (compare either the results of Model 1d to Model 1b or Model 1e to Model 1c). The fixed course control accounts for the remaining drop in coefficient size (compare either results of Models $1 \mathrm{e}$ to $1 \mathrm{~d}$ or $1 \mathrm{c}$ to $1 \mathrm{~b})$. This suggests that latent confounders primarily consist of student traits, followed by contextual course characteristics. In the Discussion section, we discuss why students' disposition of self-control (Ridder et al., 2012) is one candidate for such an unobserved confounding student trait, among others.

Finally, we note that - like the rest of our panel models - Model 1e's estimate of $\beta$ is negative and its confidence interval contains mostly negative values. This suggests that higher smartphone use in-class is associated with lower course grades, even when controlling 
for all fixed student and course traits. However, the estimated association is quite small; we see from the standardized coefficient that increasing smartphone use by one standard deviation $(=6.1 \%)$ is only associated with a 0.045 decrease in standardized grades. Moreover, the confidence interval only barely includes zero. Thus, if our results were to be interpreted as a rejection of the null hypothesis that there is no effect of screen use in-class, this conclusion is very sensitive to choice of confidence band. ${ }^{2}$

Cross-sectional model. As expected, the estimate of $\beta$ found by the cross-sectional model (Model 2) is close to the estimate found by the panel model that only controls for observed background variables (Model 1b in Table 5), and the two models have largely overlapping confidence intervals for $\beta$. Thus, the cross-sectional model estimates the regression coefficient of average smartphone use in-class as -0.099 with $(-0.142,-0.056)$ as its 95 percent confidence interval. Estimating the cross-sectional model with standardized grades and standardized average in-class smartphone use yields a regression coefficient of -0.181 for smartphone use. Thus, a one standard deviation increase $(=5.3 \%)$ in student average in-class smartphone use predicts that the student's grade average is 0.181 standard deviations lower under the cross-sectional model. In summary, the cross-sectional model reproduces existing cross-sectional studies finding a negative and moderate association between a students' in-class device use and academic performance, even when controlling for a broad range of student background variables. However, this association should not be interpreted as a substantive causal relation, since the results of our panel models suggest that such estimates are likely biased away from zero due to unobserved confounders. A full regression table for the cross-sectional model can be found in the SOM.

\section{Discussion}

Unlike previous research, the present study inspected the influence of directly recorded in-class mobile device use and academic performance across multiple courses and years for a

\footnotetext{
${ }^{2}$ The standard error of Model 1e's estimate of $\beta$ is 0.013 and thus 0 would be included in a 95.5 pct., but not in a 94.5 pct., confidence interval.
} 
large cohort of university students. When modelled as a cross-sectional analysis (Model 2) on a dataset with one observation per individual - in accordance with the existing literature we confirmed the hypothesis that higher in-class smartphone usage, averaged across courses, was associated with a substantially lower average grade, even when controlling for a broad range of observed background variables. As expected, a similar association between students' course grades and smartphone use was found on a dataset with multiple observations per individual in a model with the same set of measured control variables (Model 1b). However, when we leveraged the panel data to control for all fixed student and course characteristics (Model 1e), the magnitude of the estimated association decreased substantially, and, while remaining mostly negative, its confidence interval included zero. As already discussed in the Results section, this difference reflects that the association between grades and in-class smartphone use was confounded both by student characteristics that were not captured by our student background variables and by contextual effects from course characteristics.

Students' disposition of self-control (Ridder et al., 2012) is one possible candidate for a confounding student trait that we do not directly observe in our data. Recent studies found that - after controlling for BFI personality domains - self-control capacity explained behavioral differences in delaying immediate device use (Berger et al., 2018; Wilmer and Chein, 2016), and a recent review found that self-control positively predicts academic performance (Duckworth et al., 2019). Thus, high levels of smartphone use in-class might primarily be a signal of low self-control and not itself a substantial cause of lower academic performance. Notably, our extensive set of measured background control variables (Model 1b) did include behavioral and cognitive correlates of self control (BMI, smoking status, locus of control and personality domains). However, adding fixed effects to control for all stable student and course characteristics (Model 1e) yielded both a substantial additional increase in variance explained and a drop in the estimated coefficient of smartphone use, suggesting two candidate possibilities. First, these observed controls may function as poor proxies for the full set of latent cognitive and neurobehavioral characteristics which comprise the construct of self-control. Second, other unmeasured and/or as of yet unidentified student traits may further confound the association between in-class smartphone use and performance. 
Given the magnitude of additional variance explained and the decline in the coefficient of smartphone use by the inclusion of student and course fixed effects, it is very possible that one or more unobserved individual and contextual traits jointly influence smartphone use and academic performance. In this regard, our results also suggest that research on device use and learning should not neglect the role of contextual course-level factors that may influence learning and device use (Stokols, 2018). Research that controls for - or directly investigates - the influence of both course-specific traits (teaching style, teacher engagement, course-topic, student density, student-teacher visual access) and place-based characteristics (lighting, noise-levels, classroom size, seating arrangements, etc.) can contribute practical insights about the context of in-class smartphone use. Thus, future research on device use and performance should try to explore these psychological and contextual frontiers. To this end, panel models can provide a pragmatic tool for comparing models that control for all unobserved stable individual or contextual characteristics to models without fixed effects that only control for the limited subset of measured confounds. Contrasting the variance explained and the magnitude of drop in coefficient size between models with and without fixed effects can help to assess omitted variable bias. Our results strongly suggest that our uncertainty about not-well understood psychological and environmental mechanisms that may jointly influence smartphone use and academic performance should be considerable.

Our findings seem at odds with the randomized controlled trials which found that nonacademic device use causes demonstrably worse performance (Risko et al., 2010; Wood et al., 2012). Apart from the aforementioned concerns of external validity, one possible explanation for this discrepancy might be that students' in-class smartphone use has little to no variation across courses, making it impossible to disentangle its influence from confounding factors, e.g. self-control, in a model that includes student specific intercepts. We address this concern in the section Multicollinearity in fixed effects models in the SOM. Another explanation is that our measurement of mobile device use did not include adjacent laptop use. If students switch non-academic device use from smartphone to laptop in some courses, but not others, the student level variation of in-class smartphone use could contain noise, and such noise could potentially account for finding little or no correlation between course- 
specific smartphone use in-class and grades. However, another advantage of using course fixed effects is that it removes such noise at the aggregate level, including systematic switching between smartphones and laptops caused by teaching style, teacher quality, availability of internet, and lack of battery in afternoon classes. What is left is student idiosyncratic differences across courses which is likely to be less of a problem.

Still, one limitation of this study is that we only observed in-class device use for smartphones. As already discussed in the Measures section, another caveat is that we did not observe the specific mobile phone apps and on screen activities that constituted overall device use. An additional limitation is that our sample consisted of Danish university students that selected to participate. Some selection was internal to the student body. We can see that non-participants had a slightly lower grade average (Kassarnig et al., 2018), so our results speak to the sub-population tracked.

In conclusion, results from our panel data analysis of two years of student smartphone activity challenge the interpretation of existing empirical results on digital device use and academic outcomes. Our results suggest that there exist individual and course traits that confound the relationship between in-class device use and academic performance and that these are difficult to control for with salient background covariates. Critically, our results indicate that controlling for all fixed individual and course factors reduces the estimated negative effect of in-class smartphone use on academic performance by almost two-thirds (see Table 5). Researchers and educators should therefore exercise caution when estimating correlations between in-class device use and academic performance from cross-sectional data, even with a rich set of controls. In particular, they should avoid making causal claims from this type of observational data. Instead, those seeking to establish causality in real-world learning settings should pursue observational or quasi-experimental studies with research designs that are more suited for robust causal inference than cross-sectional studies. These studies should ideally unobtrusively observe all of students' electronic device activity across courses and over several terms. 


\section{Author Contributions}

A. Bjerre-Nielsen developed the study concept. A. Bjerre-Nielsen and D.D. Lassen designed the study, with contributions from K. Minor and A. Andersen. Data pre-processing was performed by A. Andersen under guidance of A. Bjerre-Nielsen, analysis by A. Andersen and A. Bjerre-Nielsen, and interpretation of results by all authors. A. Andersen, A. BjerreNielsen and K. Minor drafted the manuscript and all authors provided critical revisions. All authors approved the final version of the manuscript for submission.

\section{Declaration of Conflicting Interests}

The authors declared no conflicts of interest with respect to the authorship or the publication of this article.

\section{Funding}

Research was supported by the University of Copenhagen 2016-initiative (Social Fabric), the Copenhagen Center for Social Data Science, the Center for Economic Behavior and Inequality (financed by a grant from the Danish National Research Foundation), and DISTRACT - ERC Advanced Grant project no. 834540.

\section{Acknowledgments}

We thank Robert Böhm and three anonymous referees for helpful suggestions. We thank participants at Center for Economic Behavior and Inequality for helpful discussion and comments.

\section{Open Practices Statement}

The data reported in this article was not formally preregistered. Due to compliance with rules for data processing at Statistics Denmark we are not allowed to share the dataset. Requests for access to the data can be sent via email to the corresponding author. 


\section{References}

Adelantado-Renau M, Moliner-Urdiales D, Cavero-Redondo I, Beltran-Valls MR, MartínezVizcaíno V and Álvarez-Bueno C (2019) Association between screen media use and academic performance among children and adolescents: a systematic review and metaanalysis. JAMA Pediatrics 173(11): 1058-1067.

Andrews S, Ellis DA, Shaw H and Piwek L (2015) Beyond self-report: Tools to compare estimated and real-world smartphone use. PloS one 10: e0139004.

Appel M, Marker C and Gnambs T (2019) Are social media ruining our lives? a review of meta-analytic evidence (forthcoming). Review of General Psychology .

Berger S, Wyss AM and Knoch D (2018) Low self-control capacity is associated with immediate responses to smartphone signals. Computers in Human Behavior 86: 45-51.

Brooks DC and Pomerantz J (2017) Ecar study of undergraduate students and information technology. Technical report, 2017.

Cameron AC and Miller DL (2015) A practitioner's guide to cluster-robust inference. Journal of human resources 50(2): 317-372.

Chen Q and Yan Z (2016) Does multitasking with mobile phones affect learning? a review. Computers in Human Behavior 54: 34-42.

Duckworth AL, Taxer JL, Eskreis-Winkler L, Galla BM and Gross JJ (2019) Self-control and academic achievement. Annual Review of Psychology 70(1): 373-399.

Felisoni DD and Godoi AS (2018) Cell phone usage and academic performance: An experiment. Computers \& Education 117: 175-187.

Fried C (2008) In-class laptop use and its effects on student learning. Computation E Education 50: 906-914. 
Gehlen-Baum V and Weinberger A (2014) Teaching, learning and media use in today's lectures. Computers in Human Behavior 37: 171 - 182.

Goldberg LR (1993) The structure of phenotypic personality traits. The American Psychologist 48: 26-34.

Grace-Martin M and Gay G (2001) Web browsing, mobile computing and academic performance. Educational Technology \& Society 4: 95-107.

Karpinski AC, Kirschner PA, Ozer I, Mellott JA and Ochwo P (2013) An exploration of social networking site use, multitasking, and academic performance among united states and european university students. Computers in Human Behavior 29(3): 1182 - 1192.

Kassarnig V, Bjerre-Nielsen A, Mones E, Lehmann S and Lassen DD (2017) Class attendance, peer similarity, and academic performance in a large field study. PloS one 12: $\mathrm{e} 0187078$.

Kassarnig V, Mones E, Bjerre-Nielsen A, Sapiezynski P, Lassen DD and Lehmann S (2018) Academic performance and behavioral patterns. EPJ Data Science 7: 10.

Kim I, Kim R, Kim H, Kim D, Han K, Lee PH, Mark G and Lee U (2019) Understanding smartphone usage in college classrooms: A long-term measurement study. Computers E Education : 103611.

Kirschner P and Karpinski A (2010) Facebook and academic performance. Computers in Human Behavior 26: 1237-1245.

Kraushaar J and Novak D (2010) Examining the affects of student multitasking with laptops during the lecture. Journal of Information Systems Education 21.

Lepp A, Barkley JE and Karpinski AC (2014) The relationship between cell phone use, academic performance, anxiety, and satisfaction with life in college students. Computers in Human Behavior 31: 343-350. 
McNeish D and Kelley K (2019) Fixed effects models versus mixed effects models for clustered data: Reviewing the approaches, disentangling the differences, and making recommendations. Psychological Methods 24(1): 20-35.

Mendoza JS, Pody BC, Lee S, Kim M and McDonough IM (2018) The effect of cellphones on attention and learning: The influences of time, distraction, and nomophobia. Computers in Human Behavior 86: 52-60.

Orben A, Dienlin T and Przybylski AK (2019) Social media's enduring effect on adolescent life satisfaction. Proceedings of the National Academy of Sciences 116(21): 10226-10228.

Orben A and Przybylski AK (2019) Screens, teens and psychological well-being: Evidence from three time-use diary studies. Psychological Science .

Rafaeli A, Ashtar S and Altman D (2019) Digital traces: New data, resources, and tools for psychological-science research. Current Directions in Psychological Science 28(6): $560-566$.

Ravizza S, Uitvlugt M and Fenn K (2017) Logged in and zoned out: How laptop internet use relates to classroom learning. Psychological Science 28: 171-180.

Ravizza SM, Hambrick DZ and Fenn KM (2014) Non-academic internet use in the classroom is negatively related to classroom learning regardless of intellectual ability. Computers E) Education 78: 109-114.

Ridder D, Lensvelt-Mulders G, Finkenauer C, Stok M and Baumeister R (2012) Taking stock of self-control a meta-analysis of how trait self-control relates to a wide range of behaviors. Personality and Social Psychology Review 16: 76-99. DOI: $10.1177 / 1088868311418749$.

Risko E, Buchanan D, Medimorec S and Kingstone A (2010) Everyday attention: Mind wandering and computer use during lectures. Computers in Human Behavior 68: 275283. 
Roth B, Becker N, Romeyke S, Schäfer S, Domnick F and Spinath FM (2015) Intelligence and school grades: A meta-analysis. Intelligence 53: 118-137.

Rotter JB (1993) Generalized expectancies for internal versus external control of reinforcement. Psychological Monographs: General and Applied 80: 1-28.

Stokols D (2018) Social ecology in the Digital Age: Solving complex problems in a globalized world. Academic Press.

Stopczynski A, Sekara V, Sapiezynski P, Cuttone A, Madsen MM, Larsen JE and Lehmann S (2014) Measuring large-scale social networks with high resolution. PloS one 9: e95978.

Uncapher MR and Wagner AD (2018) Minds and brains of media multitaskers: Current findings and future directions. Proceedings of the National Academy of Sciences 115(40): 9889-9896.

Uzun AM and Kilis S (2019) Does persistent involvement in media and technology lead to lower academic performance? evaluating media and technology use in relation to multitasking, self-regulation and academic performance. Computers in Human Behavior 90: 196-203.

Whitlock J and Masur PK (2019) Disentangling the association of screen time with developmental outcomes and well-being: Problems, challenges, and opportunities. JAMA Pediatrics 173(11): 1021-1022.

Wilmer HH and Chein JM (2016) Mobile technology habits: patterns of association among device usage, intertemporal preference, impulse control, and reward sensitivity. Psychonomic Bulletin \&S Review 23(5): 1607-1614.

Wilmer HH, Sherman LE and Chein JM (2017) Smartphones and cognition: A review of research exploring the links between mobile technology habits and cognitive functioning. Frontiers in Psychology 8: 605. 
Wood E, Zivcakova L, Gentile P, Archer K, De Pasquale D and Nosko A (2012) Examining the impact of off-task multi-tasking with technology on real-time classroom learning. Computers \&3 Education 58: 365-374.

Wooldridge JM (2010) Econometric analysis of cross section and panel data. 2nd edition. Cambridge and London: MIT Press. 